INPLASY

PROTOCOL

To cite: Xiong et al.

Postoperative analgesic effectiveness of quadratus lumborum block for hip surgery: a systematic review and meta-analysis. Inplasy protocol 202180063. doi: 10.37766/inplasy2021.8.0063

Received: 15 August 2021

Published: 15 August 2021

Corresponding author:

Fuming Wang

xhl319@126.com

Author Affiliation:

Chongqing General Hospital, University of Chinese

Academy of Sciences.

Support: No.

Review Stage at time of this submission: Preliminary searches.

Conflicts of interest: None declared.

\section{Postoperative analgesic effectiveness of quadratus lumborum block for hip surgery: a systematic review and meta-analysis}

Xiong, HL; Zhu, WX2; Qiao, X33 Xu, QQ4; Yang, WK5; Wang, FM6.

Review question / Objective: The goal of the present systematic review is to determine the efficacy of the quadratus lumborum block in providing postoperative analgesia for hip surgery when compared with placebo or other analgesic techniques.

Condition being studied: The quadratus lumborum block is a relatively recent technique, first described by Blanco in 2007. There has been a series of new evidence regarding the various quadratus lumborum block anatomic techniques and their effectiveness in preventing pain during hip surgery. This systematic review aims to perform a comprehensive analysis of the available randomized controlled trials to determine the efficacy of the quadratus lumborum block in providing postoperative analgesia for hip surgery compared with placebo or other analgesic techniques in adult patients.

INPLASY registration number: This protocol was registered with the International Platform of Registered Systematic Review and Meta-Analysis Protocols (INPLASY) on 15 August 2021 and was last updated on 15 August 2021 (registration number INPLASY202180063).

\section{INTRODUCTION}

Review question / Objective: The goal of the present systematic review is to determine the efficacy of the quadratus lumborum block in providing postoperative analgesia for hip surgery when compared with placebo or other analgesic techniques.

Condition being studied: The quadratus lumborum block is a relatively recent technique, first described by Blanco in 2007. There has been a series of new 
evidence regarding the various quadratus lumborum block anatomic techniques and their effectiveness in preventing pain during hip surgery. This systematic review aims to perform a comprehensive analysis of the available randomized controlled trials to determine the efficacy of the quadratus lumborum block in providing postoperative analgesia for hip surgery compared with placebo or other analgesic techniques in adult patients.

\section{METHODS}

Participant or population: Patients aged at least 18 years undergoing hip surgery.

Intervention: Quadratus lumborum block will be the main intervention.

Comparator: Placebo or other analgesic techniques will be comparators.

Study designs to be included: Randomized control trials (RCTs) will be included.

Eligibility criteria: We included randomised controlled trials (RCTs) that investigated the postoperative analgesic properties of quadratus lumborum block compared with any control in patients aged at least 18 years undergoing hip surgery.

Information sources: The following electronic databases were used: MEDLINE (PubMed), EMBASE (Elsevier), Web of Science, and Cochrane Central Registry of Controlled Trials (Wiley).

Main outcome(s): Main outcomes will include postoperative pain scores and 24hr postoperative opioid consumption.

Quality assessment / Risk of bias analysis: Grading of Recommendations Assessment, Development, and Evaluation (GRADE) will be used to assess the quality of evidence, which is classified as high, moderate, low or very low. Assessments include the risk of bias, inconsistency, indirectness, impreciseness and other considerations. The Cochrane Collaboration's tool will be used to assess the risk of bias.
Strategy of data synthesis: The metaanalysis will be performed post-hoc when an adequate number of sufficiently homogeneous studies are found after data extraction, using Review Manager software. Aggregate-level data will be used for meta-analysis. Data will be pooled using the random-effects model. Continuous outcomes measured on the same scale will be synthesized using mean difference and reported as a mean difference with $95 \%$ confidence interval (CI). Different opioids will be converted to oral morphine equivalent (OME) for comparison between the studies. Similarly, dichotomous outcomes will be reported as risk ratios with $95 \% \mathrm{Cl}$.

Subgroup analysis: We will consider subgroups such as comparator types and anatomical variations of the quadratus lumborum block.

Sensitivity analysis: Sensitivity and specificity will be estimated using hierarchical models.

Country(ies) involved: China.

Keywords: Quadratus Lumborum block; Pain outcome measurement; Hip surgery.

Contributions of each author:

Author 1 - Haolan Xiong.

Author 2 - Wenxiu Zhu.

Author 3 - Xin Qiao.

Author 4 - Qingqin Xu.

Author 5 - Wuke Yang.

Author 6 - Fuming Wang. 